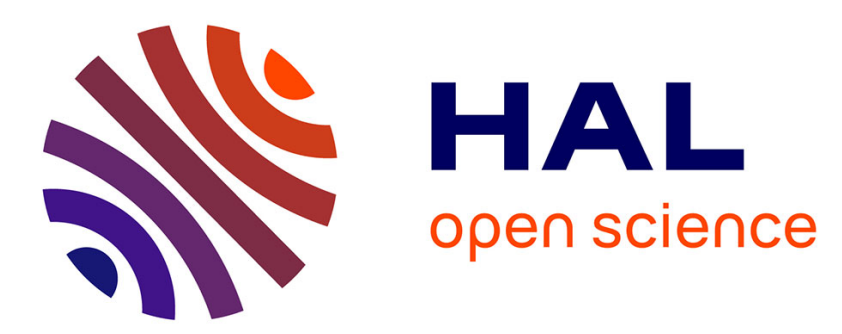

\title{
Comparison of passive inductor designs for piezoelectric shunt damping
}

Boris Lossouarn, Olivier Thierry, Mathieu Aucejo, Jean-François Deü

\section{To cite this version:}

Boris Lossouarn, Olivier Thierry, Mathieu Aucejo, Jean-François Deü. Comparison of passive inductor designs for piezoelectric shunt damping. SPIE Smart Structures/NDE 2016, Mar 2016, Las Vegas, Nevada, United States. 10.1117/12.2219200 . hal-01739220

\section{HAL Id: hal-01739220 \\ https://hal.science/hal-01739220}

Submitted on 21 Mar 2018

HAL is a multi-disciplinary open access archive for the deposit and dissemination of scientific research documents, whether they are published or not. The documents may come from teaching and research institutions in France or abroad, or from public or private research centers.
L'archive ouverte pluridisciplinaire HAL, est destinée au dépôt et à la diffusion de documents scientifiques de niveau recherche, publiés ou non, émanant des établissements d'enseignement et de recherche français ou étrangers, des laboratoires publics ou privés. 


\title{
Comparison of passive inductor designs for piezoelectric shunt damping
}

\author{
Boris Lossouarn ${ }^{\mathrm{a}}$, Olivier Thierry ${ }^{\mathrm{a}}$, Mathieu Aucejo ${ }^{\mathrm{a}}$, and Jean-François Deü ${ }^{\mathrm{a}}$ \\ ${ }^{a}$ Conservatoire National des Arts et Métiers, 2 Rue Conté, Paris, France
}

\begin{abstract}
Considering piezoelectric damping, a resonant shunt can lead to a significant vibration reduction when tuned to the mechanical mode to control. However, limits appear when looking at practical applications in a low frequency range: the required inductance is often too high to be satisfied with standard passive components. Moreover, even if the inductor is eventually available, the internal resistance of the component generally exceeds the value which is required for a shunt optimization. Suitable inductors can be designed for applications requiring high inductance and low resistance values. Indeed, the permeance of a magnetic circuit can be significantly increased by the use of closed cores made of high permeability materials. In this paper, three designs are described and compared: an inductor from standard series and two handmade inductors involving a ferrite core and a nanocrystalline toroid. The components are successively integrated into a piezoelectric shunt dedicated to the vibration control of a cantilever beam. Depending on the frequency of the target mechanical mode to control, the benefits and the limits of the different inductors are observed. It is shown that custom designs can definitely extend to lower frequency the application of the passive resonant shunt strategy.
\end{abstract}

Keywords: inductor design, resonant shunt, piezoelectricity, passive damping, closed magnetic cores

\section{INTRODUCTION}

Structural damping occurs when shunting piezoelectric material with passive electrical circuits. Resistive and resonant shunts were described by Hagood and von Flotow ${ }^{1}$ in 1991. The mechanical energy of a vibrating structure is converted by a piezoelectric patch into electrical energy, which is then dissipated into a resistor. The resonant shunt requires an inductor that is combined to the piezoelectric capacitance in order to generate an electrical resonance. Similarly to what is observed with a tuned mass damper, ${ }^{2}$ the electrical resonance enhances the energy transfer when tuned to the mechanical mode to control. Consequently, a passive and potentially lightweight solution can provide significant vibration reduction without strong modification of the mechanical structure. This control strategy was firstly applied to beams with single-mode shunts ${ }^{1,3}$ or multi-mode shunts ${ }^{4,5}$ and it was then extended to arrays of piezoelectric patches on beams ${ }^{6-8}$ or plates. ${ }^{9,10}$

A drawback of the resonant shunt technique is that practical applications generally requires large inductance values. The notion of "large inductance" is not clearly defined in the shunt damping literature because, depending on the authors, it can be in a range of $0.1 \mathrm{H}, 10 \mathrm{H}$ or even $1000 \mathrm{H} \cdot{ }^{3,8,11} \mathrm{In}$ any case, the standard inductor series are usually limited to $0.5 \mathrm{H}$, which cannot satisfy most of the resonant shunt applications. Fleming et al. ${ }^{11}$ proposed to use additional capacitance across the electrodes of the piezoelectric patches in order to decrease the required inductance. However, they also show that this solution induces a reduction of the damping performance. Another solution was proposed by Mokrani et al., ${ }^{12}$ who focus on an array of patches for the control of a rotationally periodic structure. The piezoelectric capacitance of the shunt is actually increased by suitable parallel connections. The main limit is that the whole piezoelectric network is designed for the control of a single pair of modes. Even if the inductance requirement can eventually be reduced, standard inductors present another limit that is rarely highlighted. Indeed, they offer an internal resistance which is usually too large for

Further author information: (Send correspondence to B.L.)

B.L.: E-mail: boris.lossouarn@cnam.fr, Telephone: +33140272328

O.T.: E-mail: olivier.thierry@cnam.fr, Telephone: +33140272328

M.A.: E-mail: mathieu.aucejo@cnam.fr, Telephone: +33158808581

J.F.D.: E-mail: jean-francois.deu@cnam.fr, Telephone: +33140272760 
resonant shunt applications. ${ }^{7}$ The challenging inductance and resistance requirements explain why most of the experimental validations involving resonant shunts are performed with synthetic inductors. ${ }^{3,4,6-10}$ The use of those active circuits pushes back the limits of physical inductors but it questions the implementation of purely passive resonant shunt damping.

A passive inductor is commonly made of a coil of conductive wire wound around a magnetic circuit. Contrary to what was sometimes stated, ${ }^{11,12}$ passive inductors above $1 \mathrm{H}$ are easily feasible with closed magnetic cores. ${ }^{13,14}$ Moreover, the direct equivalence between large inductance and large weight or volume ${ }^{7,9,10}$ is unfunded because the selection of a magnetic core depends on the current flowing into the component. Ferrite cores are easily available and give access to large inductance values. ${ }^{14,15}$ Furthermore, numerous magnetic materials with larger permeabilities are also available. For example, nanocrystalline alloys exhibit permeabilities up to ten times the highest ferrite permeabilities. The required inductance can then be obtained with a fewer number of turns, which reduces the internal resistance of the component. The use of closed magnetic circuits with high permeability materials thus enables the design of inductors with large inductance values and high quality factors. ${ }^{15}$ This allows the application of piezoelectric damping strategies with purely passive components. ${ }^{16,17}$

The objective of the present work is not to claim novel results related to inductor design but to proves that closed core inductors are of great interest for piezoelectric shunt applications. A first section describes the main characteristics of passive inductors and it explains how to select a suitable magnetic core from resonant shunt specifications. Suitable electrical models are also given in order to better understand the frequency dependence that is observed with passive components. A second section is then devoted to the design of inductors for the control of a cantilever beam with a pair of piezoelectric patches. A ferrite core and a nanocrystalline toroid are selected and the resulting components are compared to a standard inductor. The limits of the three designs are observed by considering their ability to damp the first three bending modes of the cantilever beam.

\section{INDUCTOR DESIGN}

The basics of inductors are presented by considering the classical equations governing magnetic circuits. The inductance is defined from the properties of the magnetic core, which is selected in order to satisfy electrical specifications. An electrical model of the component is then presented with both series and parallel resistances.

\subsection{Basics of electromagnetism}

When considering a closed core made of a linear and homogeneous magnetic material of permeability $\mu$, the equations related to the magnetic flux density $B$, the magnetic field strength $H$ and the magnetic flux $\varphi$ can be written as follows:

$$
B=\mu H, \quad \varphi=B A_{e} \quad \text { and } \quad H l_{e}=N i .
$$

The constant $A_{e}$ defines the effective cross section of the magnetic core and $l_{e}$ is the effective magnetic path length. The magnetic flux $\varphi$ is caused by the current $i$ flowing into a conductor, which is wound around the core with $N$ turns. This is represented in Fig. 1(a) in a case involving a toroidal core. With other core geometries, the flux can be split in two magnetic paths, as shown in Fig. 1(b) where a EE type core ${ }^{13,14}$ is considered. In any case, a changing magnetic flux generates a voltage $u$, which is equal to the temporal derivative of the total

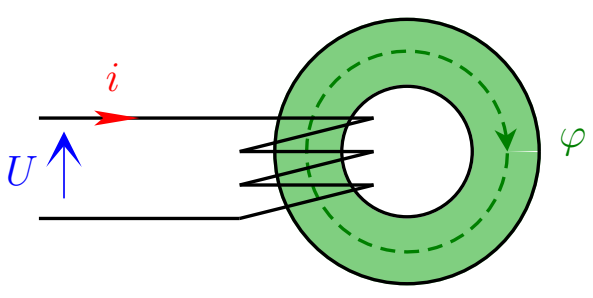

(a)

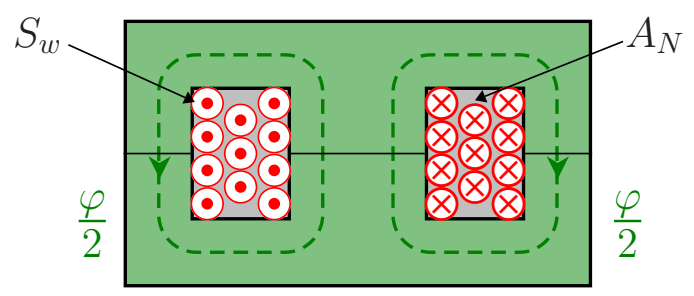

(b)

Figure 1: Two inductor designs: (a) Toroidal core. (b) EE core. 
flux going through the $N$ turns of the coil: $u=N \frac{d \varphi}{d t}$. Moreover, the inductance $L$ of an electrical circuit is defined as the ratio of the voltage to the temporal derivative of the current, i.e. $u=L \frac{d i}{d t}$. As a consequence, the inductance can be expressed from Eq. (1) by

$$
L=A_{L} N^{2} \quad \text { where } \quad A_{L}=\mu_{e} \mu_{0} \frac{A_{e}}{l_{e}}
$$

The permeability $\mu$ is here defined as the product of the vacuum permeability $\mu_{0}$ times the relative effective permeability $\mu_{e}$, which is given by the core manufacturers together with the constants $A_{e}$ and $l_{e}$. The permeance, or inductance for one turn, $A_{L}$, is thus easily calculated and can even be directly found in most of the core specification documents.

\subsection{Magnetic core selection}

In piezoelectric damping applications involving resonant shunts, an optimal inductance $L$ and an optimal equivalent series resistance $R$ are generally specified. ${ }^{1,3,4,12,18}$ Equation (2) gives the number of turns that is required to satisfy the inductance $L$ with a specific magnetic core. Naturally, the number of turns is restricted by the available room around the coil. This is observed in Fig. 1(b), where a $12^{\text {th }}$ turn could not be added because of the fixed window area $A_{N}$. This limit is quantified by the window utilization factor

$$
k_{u}=\frac{N S_{w}}{A_{N}},
$$

where $S_{w}$ is the cross section of the conductor. The factor $k_{u}$ does not usually exceed 0.5 . This can be explained by the fact that a wire with circular cross section can not entirely fill the available winding area. Another reason is the presence of insulation layers that limits the proportion of conductor area in the total wire cross section. ${ }^{13,14}$ The manufacturing process is also crucial as it determines the winding arrangements. For instance, a handmade coil with disorganized layers can lead to a significant reduction of $k_{u}$.

Once the number of turns is defined, the upper limit $k_{u}=0.5$ leads to a maximum cross section of the conductor. The geometry of the core characterizes the average length per turn $l_{N}$ and the resistance of a wire of resistivity $\rho$ is then obtained by

$$
R_{s}=\rho \frac{N l_{N}}{S_{w}}
$$

Note that an inadequate selection of the magnetic core can lead to a case where $R_{s}$ exceeds the optimal resistance $R$. In practical applications involving closed magnetic cores, we actually need to ensure $R_{s} \ll R$ because the copper loss only represents a portion of the total loss in the component. ${ }^{13-15,19}$ The introduction of a criterion for the choice of a core that is able to satisfy both specifications on $L$ and $R$ is thus crucial. Equations (2), (3) and (4) are considered and the condition on the resistance

$$
R_{s} \ll R \quad \text { gives } \quad c_{Q}=\left(\frac{k_{u} A_{L} A_{N}}{\rho l_{N}}\right) \frac{R}{L} \gg 1
$$

The criterion $c_{Q}$ is thus calculated from the core specifications and the electrical properties of the optimal shunt. The factor $k_{u}$ can be set to 0.5 and $\rho$ to $1.7 \times 10^{-8} \Omega \mathrm{m}$, which is the resistivity of copper. ${ }^{13}$ If $c_{Q}$ is below or close to 1 , the considered core cannot satisfy both $L$ and $R$ requirements. Then, another core with a larger ratio $A_{L} A_{N} / l_{N}$ would have to be found.

Two practical limits appear when considering the use of passive inductors. First, the magnetic flux density has to remain below the saturation flux density $B_{M}$ in order to avoid a strong reduction of the inductance value. The second limit concerns the current density $i / S_{w}$, which cannot exceed $J_{M}$, the maximum current density that would damage the component. From Eqs. (1), (2) and (3), we get restrictions on the effective current $i$ flowing through the conductor:

$$
i<I_{M}^{B}=\left(\frac{B_{M} A_{e}}{\sqrt{2 A_{L}}}\right) \frac{1}{\sqrt{L}} \quad \text { and } \quad i<I_{M}^{J}=\left(k_{u} J_{M} A_{N} \sqrt{A_{L}}\right) \frac{1}{\sqrt{L}}
$$

Consequently, the currents $I_{M}^{B}$ and $I_{M}^{J}$ needs to be considered as upper limits when introducing the chosen inductor into a specific electrical circuit. 


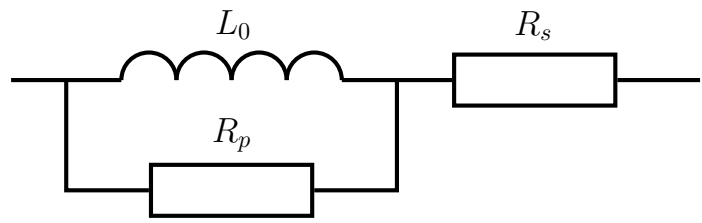

(a)

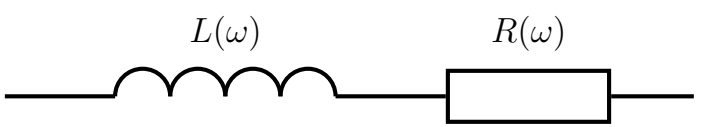

(b)

Figure 2: Electrical models for an inductor: (a) Model with a series resistance and a parallel resistance. (b) Model involving an equivalent series inductance and an equivalent series resistance

\subsection{Equivalent circuit models including resistors}

In the present application, a suitable model for the magnetic component is obtained by considering a perfect inductance $L_{0}$ combined to a resistance $R_{s}$ in series and a resistance $R_{p}$ in parallel. This electrical model is represented in Fig. 2(a). $R_{s}$ is approximated by the resistance in Eq. (4) that corresponds to the copper loss, i.e. the Joule heating in the wire. The parallel resistance $R_{p}$ represents the loss in the magnetic material, which has two main components: the hysteretic loss and the eddy current loss. ${ }^{13,14} R_{p}$ is more difficult to evaluate because it depends on the core material, on the frequency and on the flux density. For piezoelectric shunt applications involving high permeability cores of standard size, it was observed that the energy dissipation induced by $R_{p}$ is usually larger than the copper loss due to $R_{s}$. Nevertheless, the resulting equivalent series resistance is still lower than the optimal shunt resistance, as seen in the following experiments. The equivalent series resistance $R(\omega)$ is represented Fig. 2(b) together with $L(\omega)$, which is the equivalent series inductance. Both values come from the impedance of the electrical model in Fig. 2(a), which is recast in

$$
Z=R(\omega)+j \omega L(\omega) \quad \text { where } \quad R(\omega)=R_{s}+\frac{R_{p}}{1+\left(\frac{R_{p}}{L_{0} \omega}\right)^{2}} \quad \text { and } \quad L(\omega)=\frac{L_{0}}{1+\left(\frac{L_{0} \omega}{R_{p}}\right)^{2}}
$$

From this formulation, it is remarked that the equivalent series inductance and resistance necessarily depend on the frequency. This effect is due to the presence of the parallel resistance $R_{p}$ and it explains the non-negligible frequency dependence that is observed when measuring the inductance and resistance of closed core inductors.

\section{APPLICATION TO PIEZOELECTRIC SHUNT DAMPING}

Magnetic components are selected in order to damp vibration of a cantilever beam with a piezoelectric resonant shunt. It is shown that standard passive inductors cannot satisfy the inductance and resistance requirements for the lowest mode of the beam. Suitable inductors involving two different types of closed cores are thus designed and compared.

\subsection{Experimental setup}

The experimental setup consists of a cantilever beam, which is excited by a non-contact system involving a coil and a magnet at the free end of the beam. This setup was presented by Thomas et al. ${ }^{3}$ for experiments involving resistive and resonant piezoelectric shunts. The aluminum beam is $170 \mathrm{~mm}$ long, $25 \mathrm{~mm}$ wide and 2 $\mathrm{mm}$ thick and the PZT patches are $20 \mathrm{~mm}$ long, $25 \mathrm{~mm}$ wide and $0.5 \mathrm{~mm}$ thick. As seen in Fig. 3, the collocated patches are bounded near the clamping area. In the present setup, they are polarized in identical directions and connected in parallel. The velocity of the beam is measured with a laser Doppler vibrometer. The frequency response functions are then obtained by referring to the current in the coil, which is considered proportional to the input force on the frequency range of interest. ${ }^{3}$ A scanning process allows the extraction of the operating deflection shapes. Three bending modes and one torsional mode are identified below $1 \mathrm{kHz}$. They are represented in Fig. 4 with the frequency response function (FRF) related to the displacement at the free end of the beam. The torsional resonance is hardly observable on the FRF because the measurement is performed on the central axis of the beam. This is not an issue because we focus on the control of the first three bending modes. 


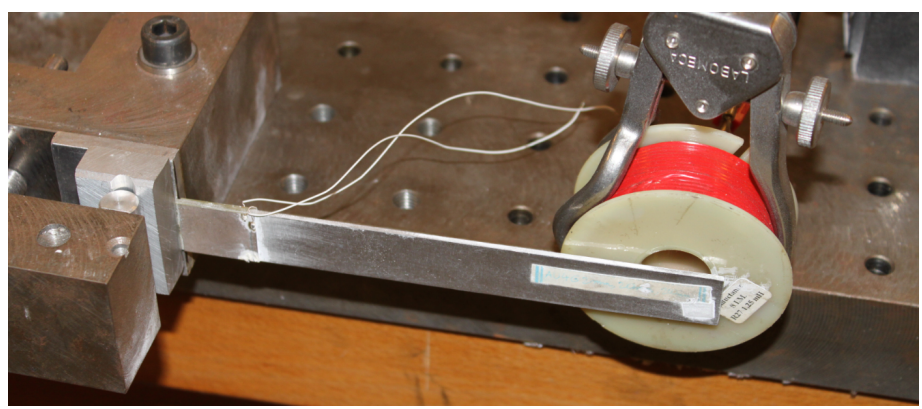

Figure 3: Experimental setup involving a cantilever beam controlled by two collocated piezoelectric patches.

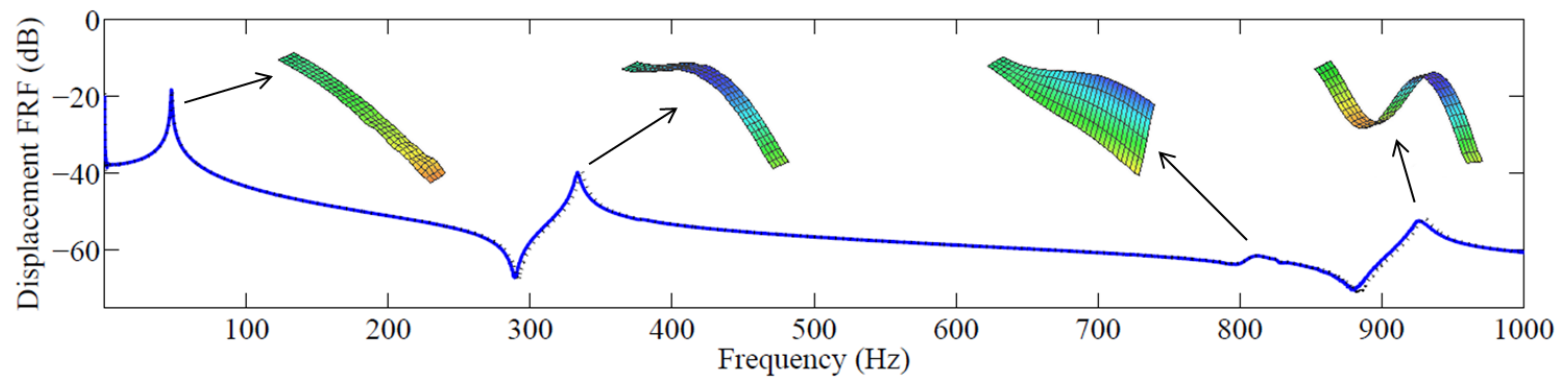

Figure 4: Displacement frequency response functions of the beam and corresponding operating deflection shapes - (-) with short-circuited patches, $(\cdots)$ with open-circuited patches.

Several methods were proposed to define the optimal inductance $L$ and resistance $R$ of a resonant shunt. ${ }^{1,3,18}$ It is remarked that they all give relatively close results when dealing with moderate values of the coupling factor. ${ }^{18}$ Here, the method proposed by Thomas et al. ${ }^{3}$ is considered, which gives

$$
L=\frac{1}{C^{\epsilon} \omega_{O}^{2}} \quad \text { and } \quad R=\sqrt{\frac{3}{2}} \frac{k_{c}}{C^{\epsilon} \omega_{O}},
$$

where $C^{\epsilon}$ is the blocked capacitance, $\omega_{O}$ is the open circuit angular frequency of the mode to control and $k_{c}$ is its coupling factor. The coupling factor is defined by $k_{c}=\sqrt{\left(\omega_{O}^{2}-\omega_{S}^{2}\right) / \omega_{S}^{2}}$, where $\omega_{S}$ is the short circuit angular frequency. The blocked capacitance is approximated by direct measurement of the piezoelectric capacitance at $100 \mathrm{~Hz}$ when no bending motion is allowed: $C^{\epsilon}=34.4 \mathrm{nF}$. From the results in Fig. 4 and Eq. (8), it then becomes possible to extract the optimal inductance and resistance for the control of the first three bending modes. The results are given in Table 1, which presents for each mode the short circuit and open circuit frequencies, $f_{S}$ and $f_{O}$, the coupling factor $k_{c}$ and the resulting series inductance $L$ and resistance $R$.

\subsection{Selection of magnetic components}

The inductance requirement for piezoelectric shunt applications is generally too high to be satisfied with standard inductors. This is illustrated by the inductance values appearing in Table 1, which all exceed the typical upper

Table 1: Piezoelectric coupling and optimal shunt specifications for the first three bending modes.

\begin{tabular}{|c|c|c|c|c|c|}
\hline & $f_{S}(\mathrm{~Hz})$ & $f_{O}(\mathrm{~Hz})$ & $k_{c}$ & $L(\mathrm{H})$ & $R(\Omega)$ \\
\hline Mode 1 & 47.07 & 47.46 & 0.129 & 330 & 15000 \\
\hline Mode 2 & 333.6 & 336.1 & 0.123 & 6.5 & 2100 \\
\hline Mode 3 & 925.8 & 930.5 & 0.101 & 0.85 & 610 \\
\hline
\end{tabular}


Table 2: Characteristics of the two magnetic cores.

\begin{tabular}{|c|c|c|c|c|c|c|c|c|}
\hline & $m(\mathrm{~g})$ & $\mu_{e}$ & $A_{e}\left(\mathrm{~mm}^{2}\right)$ & $l_{e}(\mathrm{~mm})$ & $A_{L}(\mu \mathrm{H})$ & $B_{M}(\mathrm{~T})$ & $A_{N}\left(\mathrm{~mm}^{2}\right)$ & $l_{N}(\mathrm{~mm})$ \\
\hline Ferrite core & 22 & 5720 & 98 & 44 & 16 & 0.43 & 41 & 52 \\
\hline Nanocrystalline toroid & 23 & 87500 & 40 & 79 & 56 & 1.20 & 236 & 50 \\
\hline
\end{tabular}

limit offered by most of the inductor suppliers: $0.5 \mathrm{H}$. The inductance for the third mode can be eventually satisfied with the series combination of two standard inductors. However, the control of the first two modes would require numerous components, which would largely exceed the optimal resistance. Actually, standard inductors are generally made of copper wire wound around a ferrite cylinder. About half of the magnetic circuit is thus the air where the magnetic field has to spread from one end of the cylinder to the other end. The benefit is that the air offers a relatively constant permeability, which limits the nonlinearities of the inductor. But the main drawback is the poor permeability of the air that induces low quality factors and limited inductance for standard size components. Other inductor designs that could satisfy the shunt specifications are then required.

Closed magnetic cores can offer significant permeance $A_{L}$ because the magnetic field do not has to loop through the air. This means that for a specific inductance requirement, the number of turns is considerably reduced when compared to classical ferrite cylinder designs. Then, the total resistance of the wire decreases because of the shorter length of wire and because of the eventual increase of its diameter. As a consequence, closed magnetic cores enable the design of inductors with both low series resistance and high inductance. Two closed cores of similar mass $m$ but different shapes and materials are selected. The first core is a type RM ferrite, which is described in Table 2. In term of geometry, the RM core is rather similar to the classical EE core represented in Fig. 1(b). As various shapes and materials are available for this range of components, the ferrite core providing the larger $A_{L}$ value was selected. Nevertheless, the equivalent permeability of the chosen ferrite material is considerably lower than the permeability of some nanocrystalline materials. ${ }^{13,14}$ The second core that is considered is thus a nanocrystalline toroid of $30 \mathrm{~mm}$ outside diameter, which offers a larger permeance $A_{L}$ when compared to the selected ferrite core.

The selection of the two magnetic cores is validated by the calculation of the criterion $c_{Q}$ for the three bending modes, from Eq. (5) and Tables 1 and 2. $k_{u}=0.5$ is initially set to 0.5 in order to consider a full winding area. The results are presented in Table 3 for the ferrite core and in Table 4 for the nanocrystalline toroid. Note that $c_{Q} \gg 1$, which means that both cores can satisfy the $L$ and $R$ specifications if the contribution of the parallel resistance presented in Eq. (7) is limited. The number of turns is then computed from Eq. (2) and a standard wire diameter $\Phi_{w}=2 \sqrt{S_{w} / \pi}$ is chosen by updating $k_{u}$ and ensuring its value remains below 0.5 . At the end, the maximum currents related to both conditions on the saturation flux density and on the maximum current density are obtained from Eq. (6), where $J_{M}$ is set to $3 \mathrm{~A} / \mathrm{mm}^{2} .{ }^{14}$ For the six inductor designs, the maximum current $I_{M}^{B}$ is clearly lower than $I_{M}^{J}$. In other words, an increase of the current would detune the resonant shunt before overheating the coil. This may be another advantage of the closed core inductors that would ensure the integrity of the electrical components.

The ferrite core reaches its limits when focusing on the first mode of the beam. Table 3 gives a solution with 4520 turns of 5/100 copper wire but its practical implementation is tough. Indeed, such a number of turns with a thin and thus delicate wire would require dedicated tools that were not available for the present application.

Table 3: Inductor design characteristics with the ferrite core.

\begin{tabular}{|c|c|c|c|c|c|c|}
\hline & $c_{Q}$ & $N$ & $\Phi_{w}(\mathrm{~mm})$ & $k_{u}$ & $I_{M}^{B}(\mathrm{~mA})$ & $I_{M}^{J}(\mathrm{~mA})$ \\
\hline Mode 1 & 17 & 4520 & $5 / 100$ & 0.22 & 0.41 & 5.9 \\
\hline Mode 2 & 118 & 638 & $2 / 10$ & 0.49 & 2.9 & 94 \\
\hline Mode 3 & 268 & 231 & $3 / 10$ & 0.40 & 8.1 & 210 \\
\hline
\end{tabular}


Table 4: Inductor design characteristics with the nanocrystalline toroid.

\begin{tabular}{|c|c|c|c|c|c|c|}
\hline & $c_{Q}$ & $N$ & $\Phi_{w}(\mathrm{~mm})$ & $k_{u}$ & $I_{M}^{B}(\mathrm{~mA})$ & $I_{M}^{J}(\mathrm{~mA})$ \\
\hline Mode 1 & 1366 & 2416 & $2 / 10$ & 0.32 & 0.25 & 94 \\
\hline Mode 2 & 2466 & 341 & $5 / 10$ & 0.28 & 1.8 & 590 \\
\hline Mode 3 & 5616 & 123 & $8 / 10$ & 0.26 & 4.9 & 1500 \\
\hline
\end{tabular}

This example shows that ferrite cores are not enough when considering low frequency applications involving resonant shunts. Cores with larger $c_{Q}$ ratio are then necessary. Finally, the control of the first mode can only be satisfied with the nanocrystalline toroid. This core is wound by hand according to Table 4 for the first mode but also for mode 2 and mode 3. Ferrite cores are also wound for mode 2 and mode 3 but not for the first mode because of the aforementioned practical limit. Considering standard inductors, two of them are selected, $0.5 \mathrm{H}$ and $0.32 \mathrm{H}$, in order to reach $0.82 \mathrm{H}$ by series combination. The three inductor designs are presented in Fig. (5), which shows that they all offer similar dimensions.

\subsection{Damping of vibration with passive inductors}

The inductors are successively connected to the pair of piezoelectric patches in order to reduce the vibration amplitude of the beam. First, the damping of the third bending mode is targeted. The number of turns of the two handmade inductors are adjusted in order to get resonant shunts that are correctly tuned. The ferrite core is wound with 222 turns and the nanocrystalline toroid is wound with 101 turns. The slight difference with the theoretical values presented in Tables 3 and 4 is explained by the fact that the $A_{L}$ values appearing in Tables 2 are specified at $10 \mathrm{kHz}$. Yet, the permeance varies nonlinearly with respect to the frequency, as it was observed for the inductance $L(\omega)$ in Eq. (7). Over a frequency range below $10 \mathrm{kHz}$, the $A_{L}$ value given by the core manufacturer is thus a lower estimate that could be adjusted by measurement at the frequency of interest. The damping performances of the three resonant shunts are presented in Fig. 6.

Figure 6(a) shows the effect of the shunt made of two standard inductors in series. Only one local maximum is observed, which means that the electrical resistance is above its optimal value. ${ }^{1,3}$ This can be easily verified by measuring the direct current resistance of the inductor which is $1050 \Omega$, while the optimal resistance presented in Table 1 is $610 \Omega$. Consequently, even if standard inductors can satisfy the inductance requirement for the damping of the third bending mode, they offer too much resistance to allow an optimal tuning of the resonant shunt. On the contrary, the closed core inductors offer an equivalent series resistance below the optimal shunt resistance. This is presented in Figs. 6(b) and 6(c), where it is seen that an external resistance $R^{\star}$ needs to be introduced in order to reach the optimal damping configuration. For the present experiments, it is noticed that $R^{\star}$ is clearly below the optimal resistance in Table 1 . The series resistance of the wire is yet negligible, as indicated by the large values of the $c_{Q}$ criterion for the chosen cores. This means that the loss in the magnetic

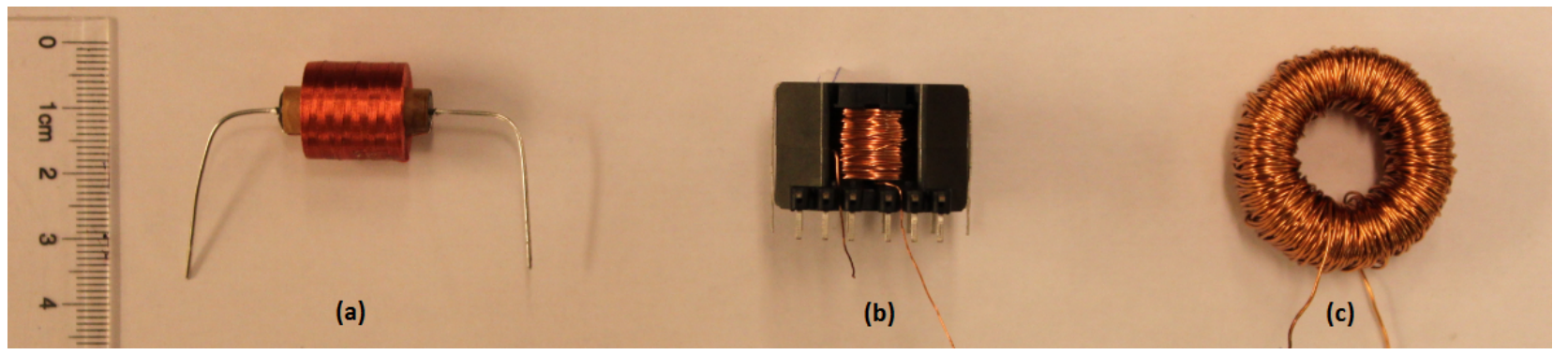

Figure 5: Three different inductor designs: (a) Standard inductor involving a ferrite cylinder. (b) Handmade inductor involving a RM ferrite core. (c) Handmade inductor involving a nanocrystalline toroid. 


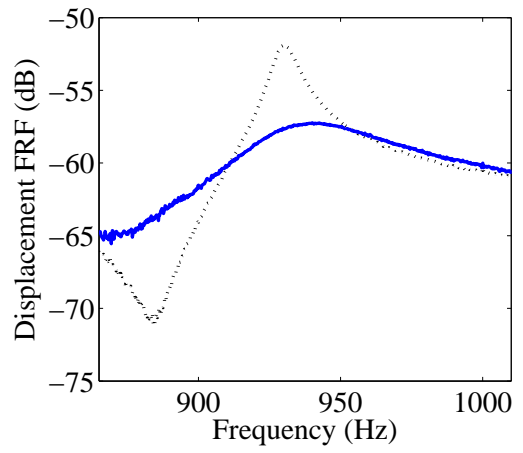

(a)

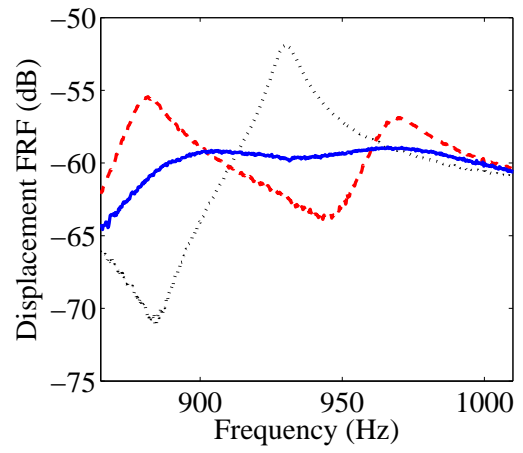

(b)

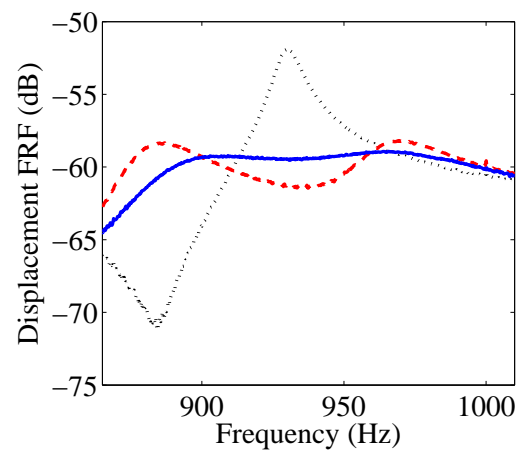

(c)

Figure 6: Damping of the third bending mode: (a) With standard inductors - ( ...) open-circuit, (-) shunted on inductor without additional resistor. (b) With the ferrite core $(N=222)-(\cdots)$ open-circuit, (- -) shunted on inductor without additional resistor, $(-)$ shunted on inductor with additional series resistor $R^{\star}=390 \Omega$. (c) With the nanocrystalline toroid $(N=101)-(\ldots)$ open-circuit, $(--)$ shunted on inductor without additional resistor, $(-)$ shunted on inductor with additional series resistor $R^{\star}=260 \Omega$.

material modeled by the parallel resistance $R_{p}$ can not be neglected. Fortunately, its influence still yields an equivalent series resistance that is lower than the optimal resistance.

The same conclusions can be drawn when focusing on the damping of the second mode. Here, the ferrite core is wound with 613 turns and the nanocrystalline toroid is wound with 296 turns. Those number of turns are still lower than the ones in Tables 3 and 4 because of the underestimated $A_{L}$ values. Furthermore, Figure 7 shows that the initially underdamped shunts can be optimized by adding series resistors $R^{\star}$. As previously, $R^{\star}$ is below the optimal series resistance because of the effect of the parallel resistance $R_{p}$.

The nanocrytalline toroid is the only core that can satisfy the $330 \mathrm{H}$ requirement for the $1^{\text {st }}$ mode of the beam. The toroid only needs 1800 turns to reach this optimal inductance, which differs from the calculated value of 2416 turns. Again, the main reason is that the equivalent permeance significantly increases when going to low frequency. Figure 8(a) shows that the handmade inductor is able to provide a significant vibration reduction.

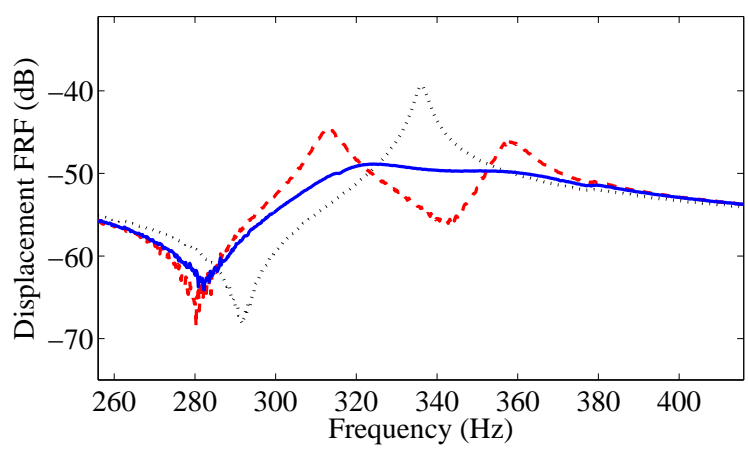

(a)

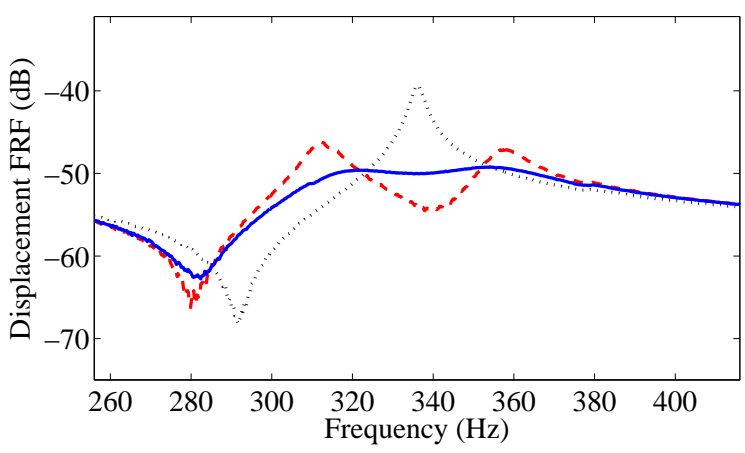

(b)

Figure 7: Damping of the second bending mode: (a) With the ferrite core $(N=613)-(\cdots)$ open-circuit, (- -) shunted on inductor without additional resistor, (-) shunted on inductor with additional series resistor $R^{\star}=1.8 \mathrm{k} \Omega$. (b) With the nanocrystalline toroid $(N=296)-(\cdots)$ open-circuit, $(--)$ shunted on inductor without additional resistor, $(-)$ shunted on inductor with additional series resistor $R^{\star}=1.4 \mathrm{k} \Omega$. 


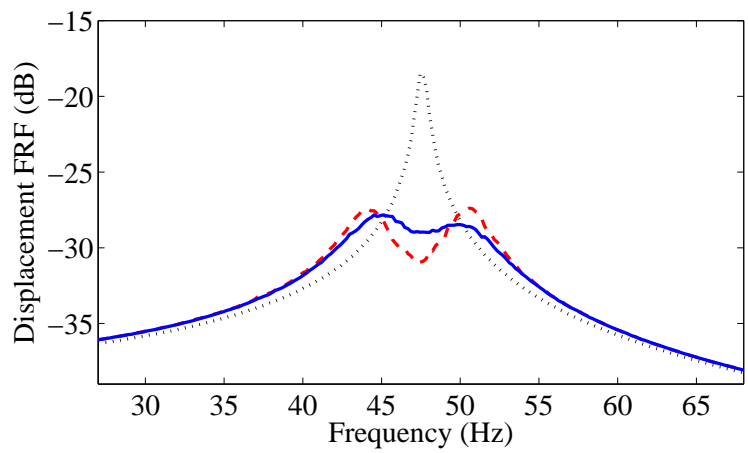

(a)

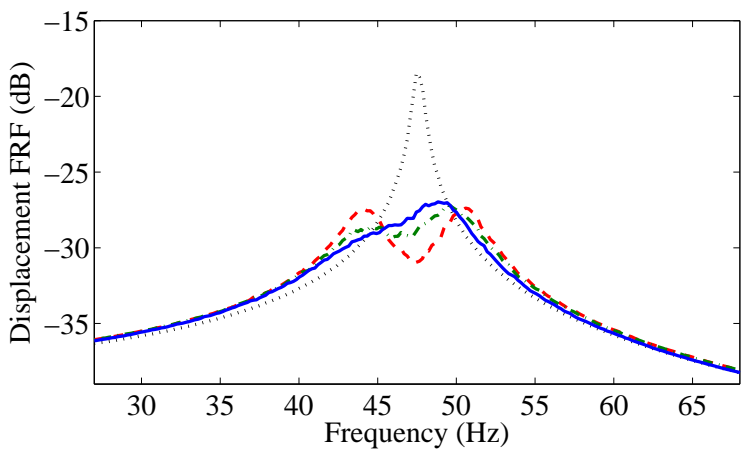

(b)

Figure 8: Damping of the first bending mode: (a) With the nanocrystalline toroid $(N=1800)-(\cdots)$ opencircuit, (- -) shunted on inductor without additional resistor, $(-)$ shunted on inductor with additional series resistor $R^{\star}=6.7 \mathrm{k} \Omega$. (b) With the nanocrystalline toroid $(N=1800)-(\cdots)$ open-circuit, (- -$)$ shunted on inductor without additional resistor at $U=1.2 \mathrm{~V},(-\cdots)$ shunted on inductor without additional resistor at $U=1.8 \mathrm{~V},(-)$ shunted on inductor without additional resistor at $U=2.4 \mathrm{~V}$.

The magnetic flux density does not reach the saturation limit because the current produced by the piezoelectric patches is only about $10 \mu \mathrm{A}$. This current is measured in the resonant shunt when the excitation amplitude causes an open-circuit displacement equal to $30 \mu \mathrm{m}$ at the end of the beam and an open-circuit voltage about $1 \mathrm{~V}$. Under this nominal excitation, the external resistance $R^{\star}$ that yields an optimal tuning of the shunt is around $6.7 \mathrm{k} \Omega$. Yet, the resistance of the wire is only $40 \Omega$ and the optimal series resistance was evaluated to $15 \mathrm{k} \Omega$ in Tables 1 . As a consequence, more than half of the energy loss occurs in the magnetic core even after the addition of the external series resistance $R^{\star}$. The energy loss in the magnetic core is modeled by the parallel resistance $R_{p}$ that appears in Fig. 2(a) and Eq. (7). The dependence of $R_{p}$ and $L$ on the amplitude of the excitation is observed experimentally. Indeed, Figure 8(b) represents the displacement FRF around the first bending mode for different excitation amplitudes. The effective voltage across the inductor is equal to $U=1.2 \mathrm{~V}$ at nominal excitation. This voltage is set to $U=1.8 \mathrm{~V}$ and then to $U=2.4 \mathrm{~V}$. The modification of the tuning is significant as it is remarked that the shunt resistance is increased and the electrical resonance moves to lower frequency. Those two effects are due to the nonlinearities of the magnetic material with respect to the excitation amplitude, ${ }^{13-15}$ which induces an increase of both the equivalent permeability and the energy loss. Those nonlinearities were also observed with the closed core inductors dedicated to mode 2 and mode 3 but the effect was less obvious. In any case, this example shows a limit of the closed magnetic cores, which are more subjected to nonlinear effects.

\subsection{Comparison of the inductor designs}

The previous inductors clearly shows that the closed magnetic cores can lead to inductance values that significantly exceed what is proposed in standard series. Ferrite cores offer decent permeabilities but some applications requires nanocrystalline materials for even larger inductance. Both solutions reduce the number of turns in the coil, which means that the copper loss can be significantly decreased. Consequently, when the loss in the magnetic core is limited, closed core inductors present considerably larger quality factors than standard inductors.

When considering the implementation of resonant shunts in industrial products, price or manufacturing process can become important issues. The standard inductors used in the present application cost around $\$ 2$ per component. The closed core designs are more expensive because a ferrite core inductor is around $\$ 5$ and the nanocrystalline toroid costs $\$ 15$. Those prices obviously depend on the number of components that is ordered but they still offer a comparison between the three designs. The standard inductors are usually cheap and offer a wide range of ready-to-use components. Yet, closed core inductors need to be specifically designed and manufactured for the considered application because suitable wound cores are not easily available. The ferrite core design offers a cylindrical coil former that can be wound by hand or with a mandrel before its introduction 
Table 5: Comparison of the three inductor designs.

\begin{tabular}{|c|c|c|c|}
\hline & Ferrite cylinder & Ferrite core & Nanocrystalline toroid \\
\hline High inductance & - & + & ++ \\
\hline Quality factor & - & ++ & ++ \\
\hline Price & ++ & + & - \\
\hline Manufacturing & ++ & + & - \\
\hline Nonlinearities & ++ & - & - \\
\hline
\end{tabular}

between the two half magnetic circuits. The geometry of the toroid makes winding harder and the automation of the process requires a specific toroidal core winding machine.

Finally, it was shown that the equivalent series inductance and resistance of the closed core inductors depend on frequency and on current amplitude. Those nonlinearities are insignificant with standard inductors where the magnetic field has to spread through the air, which offers a relatively constant permeability and low magnetic losses. This last limit of the closed core inductors also appears in Table 5, which summarizes the comparison between the three inductor designs.

\section{CONCLUSIONS}

This paper focuses on the design of passive inductors for piezoelectric shunt applications. From the basic equations describing the design of a magnetic component, a criterion based on inductance and resistance specifications is proposed in order to chose a suitable magnetic core. The electrical model with series and parallel resistances is then highlighted and related to the classical model involving the equivalent series inductance and resistance that appear in most of the shunt optimizations. The experimental setup based on a cantilever beam covered by a pair of piezoelectric patches requires inductance up to $330 \mathrm{H}$ for a control based on a passive resonant shunt. It is shown that such a high inductance value is not impractical for piezoelectric applications because of the low amplitude of the current generated by the patches. Specific designs involving closed magnetic core are required in order to reach large inductance values with low series resistance. Two magnetic cores are proposed: a RM ferrite and a nanocrystalline toroid. Both solutions can easily provide inductance above $0.5 \mathrm{H}$, which is considered as the upper limit of standard inductor series. The inductor designs are successively validated by observing significant vibration reduction for the first three bending modes of the cantilever beam. Some limits are still remarked as the nonlinearities induced by the high permeability materials. Nevertheless, closed magnetic cores clearly extends the resonant shunt strategy to lower frequencies that were not reachable with standard inductors. This solution counters the use of synthetic inductors with strictly passive components, which can be of great interest for piezoelectric shunts or even energy harvesting applications.

\section{ACKNOWLEDGMENTS}

The authors would like to acknowledge the French Ministry of National Education, Higher Education and Research for a three-year doctoral scholarship and the support of Snecma, which contributes to a Cifre thesis.

\section{REFERENCES}

[1] Hagood, N. W. and von Flotow, A., "Damping of structural vibrations with piezoelectric materials and passive electrical networks," J. Sound Vib. 146, 243-268 (1991).

[2] Den Hartog, J. P., [Mechanical vibrations], McGraw-Hill, New York (1940).

[3] Thomas, O., Ducarne, J., and Deü, J.-F., "Performance of piezoelectric shunts for vibration reduction," Smart Mater. Struct. 21, 015008 (2012).

[4] Hollkamp, J. J., "Multimodal passive vibration suppression with piezoelectric materials and resonant shunts," J. Int. Mater. Sys. Struct. 5, 49-57 (1994). 
[5] Wu, S. Y., "Method for multiple mode piezoelectric shunting with single pzt transducer for vibration control," J. Int. Mater. Sys. Struct. 9, 991-998 (1998).

[6] Airoldi, L. and Ruzzene, M., "Design of tunable acoustic metamaterials through periodic arrays of resonant shunted piezos," New J. Phys. 13, 113010 (2011).

[7] Wang, G., Chen, S., and Wen, J., "Low-frequency locally resonant band gaps induced by arrays of resonant shunts with Antoniou's circuit: experimental investigation on beams," Smart Mater. Struct. 20, 015026 (2011).

[8] Airoldi, L. and Ruzzene, M., "Wave propagation control in beams through periodic multi-branch shunts," J. Int. Mater. Sys. Struct. 22, 1567-1579 (2011).

[9] Casadei, F., Ruzzene, M., Dozio, L., and Cunefare, K. A., "Broadband vibration control through periodic arrays of resonant shunts: experimental investigation on plates," Smart Mater. Struct. 19, 015002 (2010).

[10] Chen, S., Wang, G., Wen, J., and Wen, X., "Wave propagation and attenuation in plates with periodic arrays of shunted piezo-patches," J. Sound Vib. 332, 1520-1532 (2013).

[11] Fleming, A. J., Behrens, S., and Moheimani, S. O. R., "Reducing the inductance requirements of piezoelectric shunt damping systems," Smart Mater. Struct. 12, 57 (2003).

[12] Mokrani, B., Bastaits, R., Horodinca, M., Romanescu, I., Burda, I., Viguié, R., and Preumont, A., "Parallel piezoelectric shunt damping of rotationally periodic structures," Adv. Mater. Sci. Eng. 2015 (2015).

[13] McLyman, C. W. T., [Transformer and Inductor Design Handbook], CRC Press (2004).

[14] Multon, B., "Composants passifs de l'électronique de puissance (magnétiques et capacitifs)," Lecture, https://cel.archives-ouvertes.fr/cel-01246811 (2015).

[15] Epcos, Ferrites and accessories. General - Definitions (2006).

[16] Lossouarn, B., Deü, J.-F., and Aucejo, M., "Multimodal vibration damping of a beam with a periodic array of piezoelectric patches connected to a passive electrical network," Smart Mater. Struct. 24, 115037 (2015).

[17] Lossouarn, B., Cunefare, K. A., Aucejo, M., and Deü, J.-F., "Multimodal damping of a plate with a passive piezoelectric network," Proceedings of IMAC-XXXIV (2016).

[18] Soltani, P., Kerschen, G., Tondreau, G., and Deraemaeker, A., "Piezoelectric vibration damping using resonant shunt circuits: an exact solution," Smart Mater. Struct. 23, 125014 (2014).

[19] Agilent Technologies, Agilent Impedance Measurement Handbook. A guide to measurement technology and techniques. $4^{\text {th }}$ Edition (2009). 